\title{
Geodesic Acoustic Eigenmodes
}

\author{
Kimitaka ITOH, Sanae-I. ITOH ${ }^{1)}$, Patrick H. DIAMOND ${ }^{2)}$, Akihide FUJISAWA, \\ ${\text { Masatoshi } \text { YAGI }^{1)} \text {, Tetsuo WATARI, Yoshihiko NAGASHIMA }}^{1)}$ and Atsushi FUKUYAMA ${ }^{3)}$ \\ National Institute for Fusion Science, Toki 509-5292, Japan \\ 1) Research Institute for Applied Mechanics, Kyushu University, Kasuga 816-8580, Japan \\ ${ }^{2)}$ Department of Physics, University of California San Diego, San Diego CA 92093-0319, U.S.A. \\ ${ }^{3)}$ Department of Nuclear Engineering, Kyoto University, Kyoto 606-8501, Japan
}

(Received 13 March 2006 / Accepted 10 June 2006)

The eigenmode of a geodesic acoustic mode in the presence of a temperature gradient is discussed. Eigenmodes are obtained and the characteristic wavelength scales as $\rho_{\mathrm{i}}^{2 / 3} L_{\mathrm{T}}^{1 / 3}\left(\rho_{\mathrm{i}}\right.$ : ion gyroradius, $L_{\mathrm{T}}$ : temperature gradient scale length). The direction of propagation is discussed.

(c) 2006 The Japan Society of Plasma Science and Nuclear Fusion Research

Keywords: geodesic acoustic mode, zonal flow, eigenmode, eigenfrequency, temperature gradient

DOI: $10.1585 /$ pfr. 1.037

Zonal flows have attracted attention owing to their essential role in the turbulent transport of magnetically confined plasmas [1]. The geodesic acoustic mode (GAM) is a kind of zonal flow, which has finite real frequency owing to the geodesic curvature of a toroidal magnetic field [2], and is driven by microscopic turbulence [3,4]. Measurements of GAMs have been recently reported [5-11]. It has been known that the GAMs have real frequency $\omega_{\mathrm{G}}=\sqrt{2} c_{\mathrm{s}} / R$ in tokamaks $\left(c_{\mathrm{s}}\right.$ : ion sound velocity, $R$ : majour radius). [The coefficient $\sqrt{2}$ depends on the model of plasma dynamics [1], but this is not an issue addressed in the present article.] In tokamaks and other toroidal plasmas, the plasma temperature changes in the radial direction, so that the dispersion relation $\omega=\omega_{\mathrm{G}}$, which is provided by the local theory, predicts different frequencies at different radii. In contrast, fluctuations with a common frequency are observed within a region which has a substantial width in radial direction $[10,11]$. This indicates that the GAM oscillation appears as an eigenmode. In this article, we discuss the eigenmode of GAM oscillation in the presence of a temperature gradient. Due to the finite ion gyroradius, local oscillations on different magnetic surfaces interfere with one another so as to constitute a radial eigenmode. The characteristic wavelength is found to scale as $\rho_{\mathrm{i}}^{2 / 3} L_{\mathrm{T}}^{1 / 3}\left(\rho_{\mathrm{i}}\right.$ : ion gyroradius, $L_{\mathrm{T}}$ : temperature gradient scale length) and propagates outward if the temperature decreases towards the edge.

The dispersion relation of GAMs, $\omega=\omega_{\mathrm{G}}$, is derived by balancing the cross-field current $\tilde{J}_{\mathrm{D}, r}$ (due to the magnetic field curvature) and the ion polarization current $\tilde{J}_{\mathrm{p}, r}$ under the imposition of an electrostatic perturbation that has a form $\tilde{\phi} \exp (\mathrm{i} k r-\mathrm{i} \omega t)$ in the leading order [12-15]. In order to study the radial eigenmode with analytic transparency, we take a simple collisionless limit with $T_{\mathrm{e}} \gg T_{\mathrm{i}}$

author'se-mail: itoh@nifs.ac.jp and $k \rho_{\mathrm{i}} \ll 1$. In the limit of $T_{\mathrm{e}} \gg T_{\mathrm{i}}$, the relation $v_{\text {th, } \mathrm{i}} / R \ll \omega$ holds for $\omega \sim \omega_{\mathrm{G}}$, and $\tilde{J}_{\mathrm{D}, r}$ is dominated by the electron response ( $v_{\mathrm{th}, \mathrm{i}}$ : ion thermal velocity) [14]. Therefore, $\tilde{J}_{\mathrm{D}, r}$ is not significantly influenced by the finite gyroradius effect. In contrast, the ion polarization current, which is in proportion to $\omega$, is screened by the factor $1-k^{2} \rho_{\mathrm{i}}^{2}$ owing to the finite gyroradius effect. Thus, the relation $\tilde{J}_{\mathrm{p}, r}+\tilde{J}_{\mathrm{D}, r}=0$ provides

$$
\left(1-k^{2} \rho_{\mathrm{i}}^{2}\right) \omega^{2}=\omega_{\mathrm{G}}^{2}
$$

where the lowest order finite-gyroradius correction is included (see [12-16] for a more detailed derivation). We consider the case in which the temperature decreases in radius, and choose the radius $r_{0}$ where $\omega^{2}=\omega_{\mathrm{G}}^{2}\left(r_{0}\right)$ holds. Taking the radial gradient of temperature into account, we write $\omega_{\mathrm{G}}^{2}(r)=\omega_{\mathrm{G}}^{2}\left(r_{0}\right)\left[1-\left(r-r_{0}\right) L_{\mathrm{T}}^{-1}\right]$. The dispersion relation (1) can be rewritten as an eigenmode equation

$$
\rho_{\mathrm{i}}^{2} \frac{\mathrm{d}^{2}}{\mathrm{~d} r^{2}} \phi(r)+\frac{r-r_{0}}{L_{\mathrm{T}}} \phi(r)=0,
$$

by the replacement $k^{2} \rho_{\mathrm{i}}^{2} \rightarrow-\rho_{\mathrm{i}}^{2} \mathrm{~d}^{2} / \mathrm{d} r^{2}$. Equation (2) has a characteristic scale length,

$$
\lambda=\rho_{\mathrm{i}}^{2 / 3} L_{\mathrm{T}}^{1 / 3}
$$

and is normalized as

$$
\frac{\mathrm{d}^{2}}{\mathrm{~d} x^{2}} \phi(x)+x \phi(x)=0
$$

where $x=\left(r-r_{0}\right) \lambda^{-1}$. Equation (4) is readily solved by employing the Airy function:

$$
\phi(x)=\operatorname{Ai}(-x)
$$

The result seen in Eq. (5) shows that the eigenmode peaks near the region $x \simeq 0$, propagates in the lowertemperature region $(x>0)$, and is evanescent in the higher 


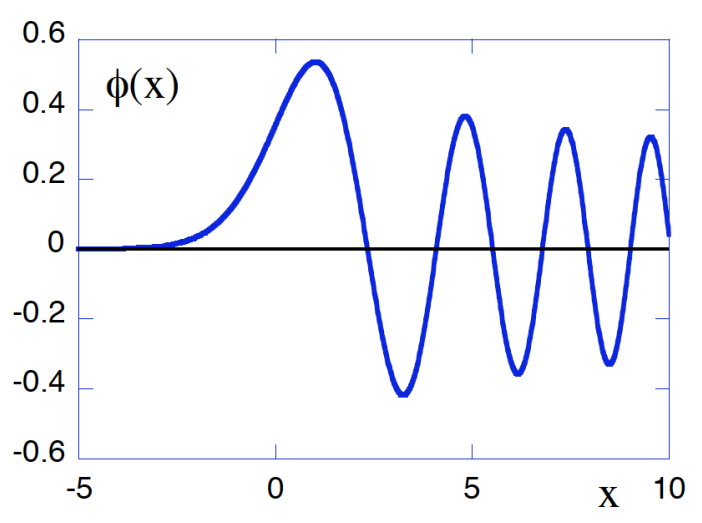

Fig. 1 GAMs radial eigenmode. Horizontal axis is normalized as $x=\left(r-r_{0}\right) \lambda^{-1}$.

temperature region $(x<0)$. Figure 1 illustrates the radial eigenfunction. The wave length is a few times $\lambda$. For this solution (5), the finite gyroradius correction has the order of magnitude $k^{2} \rho_{\mathrm{i}}^{2} \sim \rho_{\mathrm{i}}^{2 / 3} L_{\mathrm{T}}^{-2 / 3}$, and is much smaller than unity if $\rho_{\mathrm{i}} \ll L_{\mathrm{T}}$ holds. The assumption $k \rho_{\mathrm{i}} \ll 1$ is verified a posteriori. We note that, in the limit of $\rho_{\mathrm{i}} \rightarrow 0$, an eigenmode is localized to a magnetic surface.

In summary, the GAM oscillation was found to exist in a form of radial eigenmode when the temperature is inhomogeneous. This is consistent with the observation that GAM oscillations are observed as radial eigenmodes [11]. The radial wavelength has a dependence of $\rho_{\mathrm{i}}^{2 / 3} L_{\mathrm{T}}^{1 / 3}$, showing that GAMs are mesoscale fluctuations.

One can extend this analysis in a couple of ways. The extension to a more general profile of temperature $T(r)$ is possible. When $T_{\mathrm{e}}$ comes closer to $T_{\mathrm{i}}$, the screening owing to the finite-gyroradius effect also appears in $\tilde{J}_{\mathrm{D}, r}$ as was explained in [12-15], so that the coefficient to $k^{2} \rho_{\mathrm{i}}^{2}$ in Eq. (1) becomes smaller (i.e., the radial wavelength becomes shorter). As was pointed in [17], the finite ion gyroradius effect can lead to the collisionless ion damping even in the limit of $k_{\|} v_{\mathrm{th}, \mathrm{i}} \ll \omega$, such collisionless damping having recently been studied theoretically [15]. When a small but finite damping rate is introduced, the eigenfunction shows an oscillation in the region of $x<0$. Details of these investigations are left for future research.

Discussions with Dr. T. Ido, Dr. K. Hallatschek, Dr. Z. Gao, Prof. H. Sugama and Prof. H. Sanuki are greatly acknowledged. This work is partly supported by a Grantin-Aid for Scientific Research of MEXT (15360495), by a Grant-in-Aid for Specially-Promoted Research of MEXT (16002005), by the collaboration programs of NIFS (NIFS03KDAD001, NIFS03KKMD001) and the Research Institute for Applied Mechanics of Kyushu University, and by Asada Science Foundation.

[1] P.H. Diamond, S.-I. Itoh, K. Itoh and T.S. Hahm, Plasma Phys. Control. Fusion 47, R35 (2005).

[2] N. Winsor, J.L. Johnson and J.M. Dawson, Phys. Fluids 11, 2448 (1968).

[3] K. Hallatschek and D. Biskamp, Phys. Rev. Lett. 86, 1223 (2001).

[4] K. Itoh, K. Hallatschek and S.-I. Itoh, Plasma Phys. Control. Fusion 47, 451 (2005).

[5] Y. Hamada et al., Fusion Eng. Des. 34-35, 663 (1997).

[6] T. Ido et al., Nucl. Fusion 46, 512 (2006).

[7] G.R. McKee et al., Plasma Phys. Control. Fusion 45, A477 (2003).

[8] G.D. Conway et al., Plasma Phys. Control. Fusion 47, 1165 (2005).

[9] Y. Nagashima et al., Phys. Rev. Lett. 95, 095002 (2005).

[10] A. Fujisawa et al., Plasma Phys. Control. Fusion 48, S31 (2006).

[11] T. Ido et al., Plasma Phys. Control. Fusion 48, S41 (2006).

[12] V.B. Lebedev, P.N. Yushmaanov, P.H. Diamond, S.V. Novakovskii and A.I. Smolyakov, Phys. Plasmas 3, 3023 (1996).

[13] S.V. Novakovskii, C.S. Liu, R.Z. Sagdeev and M.N. Rosenbluth, Phys. Plasmas 4, 4272 (1997).

[14] T. Watari et al., Phys. Plasmas 12, 062304 (2005).

[15] H. Sugama and T-H. Watanabe, Phys. Plasmas 13, 012501 (2006).

[16] Z. Gao et al., submitted to Phys. Plasmas.

[17] H. Sanuki, Phys. Fluids 27, 2500 (1984). 